\title{
ANÁLISIS
}

\section{Empresarios y régimen de bienestar: el caso de Corea del Sur}

\section{Entrepreneurs and the Welfare Regime: The Case of South Korea}

\author{
DOI: $10.32870 /$ mycp.v10i28.722
}

\section{Resumen}

En este artículo se profundiza sobre preferencias de políticas sociales de organizaciones empresariales en Corea del Sur. Se pretende comprender si con las preferencias encontradas en estas organizaciones (en sus páginas web, en declaraciones a la prensa y en entrevista realizada por el autor de este texto) se acercan o alejan de la histórica y conservadora coalición pro-crecimiento del régimen de bienestar coreano, frente a la más reciente coalición redistributiva.

El artículo se divide en cinco partes, además de la introducción teórica e histórica: una contextual sobre desigualdad, bienestar y élites en Corea del Sur; una segunda sobre organizaciones empresariales y bienestar en Corea del Sur: la Federation of Korean Industries y la Korea Enterprises Federation; una tercera que aborda organizaciones empresariales y bienestar en Corea del Sur: economía política y protección laboral; una cuarta acerca de organizaciones empresariales y bienestar en Corea del Sur: las políticas sociales; y una quinta con las conclusiones. En el artículo se concluye que las organizaciones empresariales estudiadas continúan con las proposiciones centrales de la coalición procrecimiento, aunque sin mostrar una visión residual de las políticas sociales.

Palabras clave: Corea del Sur, organizaciones empresariales, regímenes de bienestar, políticas sociales, coaliciones promotoras.

\author{
Enrique Valencia Lomeli ${ }^{1,2}$
}

\begin{abstract}
This article explores the social policy preferences of business organizations in South Korea. It seeks to understand whether the preferences found in these organizations (on their web pages, in statements to the press, and in an interview conducted by the author of this text) are approaching or distancing themselves from the historic and conservative progrowth coalition of the Korean welfare regime, in front of the most recent redistributive coalition.

The article is divided into five parts, in addition to the theoretical and historical introduction: a contextual one on Inequality, welfare, and elites in South Korea; a second on Business organizations and welfare in South Korea: the Federation of Korean Industries and the Korea Enterprises Federation; a third, business organizations and welfare in South Korea: political economy and labor protection; a fourth, business organizations and welfare in South Korea: social policies; and a fifth with the conclusions. The article concludes that the business organizations studied continue with the central propositions of the pro-growth coalition, although without showing a residual vision of social policies.
\end{abstract}

Keywords: South Korea, business organizations, welfare regimes, social policies, advocacy coali-

tions.

Artículo recibido el 31 de agosto de 2020 y dictaminado el 27 de octubre de 2020.

1. Universidad de Guadalajara, CUCSH, Departamento de Estudios sobre Movimientos Sociales. Guanajuato \#1045, Col. Alcalde Barranquitas, C.P. 44260. Guadalajara, Jalisco, México. ORCID: https://orcid.org/0000-0003-3359-0759 Correo electrónico: enrivalo@gmail.com

2. Estoy particularmente agradecido por el apoyo técnico de Daniel Zazueta para el desarrollo de este texto y de las atinadas propuestas de Yasodhara Silva. Agradezco también los comentarios de los participantes en la mesa "Estados de Bienestar y Regímenes de Bienestar" de la XVI Conferencia de la East Asian Social Policy (EASP), celebrada en la Universidad de Taiwán en julio de 2019. 


\section{Introducción}

Los regímenes de bienestar contienen un conjunto de acuerdos para distribuir tareas de producción del bienestar entre Estado, mercado y hogares (Esping-Andersen, 1990, 1999), así como en comunidades. En el siglo XXI la polarización social ha crecido si tomamos en cuenta los indicadores de desigualdad de ingreso y de desigualdad global de la riqueza (Kim, 2018; Klasen et al., 2016; Piketty, 2013). ${ }^{3}$ Este crecimiento de la desigualdad cuestiona tanto la cohesión social como los acuerdos centrales de los regímenes de bienestar, porque limita los compromisos de construcción de bienestar de las empresas y los Estados, y deja más tareas a los hogares y a las comunidades (indígenas en el caso de varios países latinoamericanos con significativa heterogeneidad cultural). En este contexto de polarización, con crecimiento de las desigualdades en la riqueza y en varios casos de las desigualdades de ingreso, ¿cuáles son las posiciones de los diversos actores sociales? ¿Cómo se sitúan ellos mismos frente a las diversas posibilidades de confrontar las desigualdades, especialmente a través de los regímenes de bienestar? ¿Qué proponen y a qué se oponen? ¿Cuáles son sus preferencias en términos de políticas de bienestar?

En la trayectoria de los regímenes de bienestar se condensan espacios de conflictividad o de confrontación entre diversos proyectos, espacios de acuerdos generales o parciales, de resistencias; en ella se concentran propuestas de cambio (y sus alternativas o resistencias) sustentadas por actores sociales diversos, generalmente coaligados (Sabatier \& Weible, 2007). Estas disputas "se expresan especialmente en la formación de políticas e instituciones sociales, que a fin de cuentas no solamente implican al Estado sino también la definición de lo que en cada sociedad se concibe como obligaciones estatales, de los hogares, del mercado y de las comunidades"; la creación de las políticas sociales, la identificación pública de lo que se considera problema social y las instituciones sociales, se convierten en "arenas en las que se definen las aportaciones (responsabilidades) públicas, mercantiles, familiares y comunitarias" (Valencia, 2019, p. 167).

3. Solo algunas sociedades, en los primeros años del siglo escaparon a este incremento de las desigualdades (véanse por ejemplo: Boyer, 2014; y Cornia, 2016, en relación con algunas naciones latinoamericanas). 
Por consiguiente, es de particular interés en los estudios comparativos de regímenes de bienestar analizar, desde la perspectiva de la creación de coaliciones promotoras a ciertas políticas sociales (Sabatier \& Weible, 2007), las diversas posiciones de los empresarios y sus asociaciones, y de conglomerados, acerca de las tareas de bienestar en general, de las políticas sociales en particular y de los factores que, en su percepción, explican la pobreza y la desigualdad. Otros trabajos ${ }^{4}$ han analizado la participación de diversos actores civiles, sindicales y estatales en Corea del Sur; ${ }^{5}$ aquí nuestro interés es analizar las perspectivas empresariales frente a las instituciones del bienestar en este país, en el contexto de las advocacy coalitions en las políticas sociales de este país.

Los debates sobre la caracterización del régimen de bienestar coreano se han ido incrementando. Hay cierto acuerdo en señalar que en los años sesenta y setenta, años de fuerte crecimiento económico, este régimen tenía un fuerte contenido minimalista y residual (Shin, 2003), que dejaba un papel central en el compromiso de las familias en el bienestar y muy poca presencia estatal en instituciones sociales, y resistencias empresariales para la creación de instituciones sociales. ${ }^{6}$ Se fueron generando instituciones del bienestar en los años setenta y ochenta, pero de acuerdo con diversos autores, con una característica "productivista" en el régimen de bienestar en el que las políticas sociales estaban subordinadas a los objetivos económicos (Holliday, 2005), sin autonomía de las instituciones sociales.

Impulsado el proceso de democratización en 1987 y generados fuertes problemas económicos y sociales en la crisis financiera de 1997, el régimen de bienestar coreano fue cambiando en medio de pugnas entre diversas coaliciones (véase Fiori \& Kim, 2011). En Valencia (2019, pp. 191 y 192) se muestran históricamente dos coaliciones en pugna en la construcción del régimen de bienestar coreano. Resume la confrontación de la siguiente manera:

El dinamismo económico, la generación de empleos y la industrialización exitosa permitieron la construcción de una poderosa coalición social procrecimiento (militares, grandes conglomerados, sindicatos corporativos y clases medias nacientes); crecimiento primero, instituciones del bienestar después, era la síntesis de la regla defendida y promovida. (...) Sin embargo, junto con los procesos democ-

4. Por ejemplo Fiori y Kim (2011), Fleckenstein y Lee (2017), y Valencia (2019).

5. De aquí en adelante nos referiremos a Corea del Sur sólo como Corea, excepto en los títulos.

6. En especial en lo que se refiere al sistema de pensiones (Shin, 2003, p. 96). 
ratizadores fueron surgiendo actores sociales que proponían nuevas maneras de organizar la división del trabajo para el bienestar: en síntesis, más compromiso del Estado en la generación de instituciones de corte universal para enfrentar los riesgos de pobreza, vejez y enfermedades. Se fue creando así una coalición probienestar o redistributiva. El valor prioritario del dinamismo económico era desafiado por los valores de la redistribución, la equidad, el bienestar, el derecho a una vida digna (básicos del bienestar).

En este marco, ya en los años 2000 el régimen de bienestar coreano ha dejado atrás su inicial carácter residual y ha ido más allá de los elementos productivistas. Diversos autores acentúan el avance de características universalistas en el régimen coreano. Shin (2003, p. 185) subraya la adopción de un "sistema de bienestar más redistributivo", de manera semejante a Sun (2002), Valencia (2009) y Fleckenstein y Lee (2017) que destacan ya sea la generación de política de bienestar de corte socialdemócrata, ya sea simplemente universalistas.

Una tarea pendiente es incorporar las perspectivas empresariales en la tarea cotidiana por modelar recientemente el régimen de bienestar e indagar cómo actores empresariales buscan defender sus intereses en "los modos en que se reparte la producción de bienestar entre el Estado, el mercado y las familias" (Esping-Andersen, 1999, p. 73). Nos parece que es necesario profundizar acerca de las preferencias de políticas de bienestar de los organismos empresariales más relevantes, actores centrales en las relaciones de poder y de construcción del bienestar. Probablemente se dio más importancia al papel de los empresarios (sobre todo los grandes conglomerados) en la generación de un régimen de bienestar productivista, en la etapa previa a la democratización, que en las resistencias a los cambios posteriores que apuntan hacia características universalistas en el régimen de bienestar coreano (Fleckenstein \& Lee, 2017). ${ }^{7}$ Por ello, en este artículo serán analizadas las posiciones de organismos empresariales de Corea en relación con las políticas sociales en el periodo 2009 a $2019 .^{8}$

7. Puede verse en Valencia $(2009,2019)$ una revisión más detallada de trabajos académicos alrededor de los cambios recientes en el régimen de bienestar coreano.

8. El criterio de selección de este periodo es incluir los debates en torno a las políticas sociales a partir del año 2009, de fuerte crisis global y coreana, hasta 2019. En periodos de crisis y poscrisis se profundizan generalmente los debates sobre alternativas sociales para afrontar los problemas, lo que permite observar diferentes posicionamientos. La ventaja además es que este periodo incluye tres gobiernos con diferente orientación ideológica, como se verá más adelante. 


\section{Desigualdad, bienestar y élites en Corea del Sur}

Un primer camino introductorio puede ser a través de encuestas de percepción, camino con dificultades ante la ausencia (o nuestro desconocimiento) de encuestas en las que sean entrevistados un número representativo de empresarios. Sin embargo, existe un conjunto de encuestas que nos pueden permitir acercamientos iniciales a estas temáticas con las que nos aproximemos a la discusión que nos interesa. Para ello vamos a utilizar dos encuestas, con levantamientos en Corea en nuestro periodo de interés. La primera es la Encuesta de Valores con levantamiento en Corea en 2010; la segunda es la Encuesta International Social Survey Programme (ISSP) con levantamiento en este país en 2009. Trabajaremos en ellas como un primer acercamiento descriptivo acerca de las percepciones de las élites socioeconómicas coreanas.

En una primera aproximación pareciera que en Corea domina una visión crítica de la desigualdad, que aquí denominaremos redistributiva (véase Tabla 1): $90 \%$ de los encuestados considera muy grande la diferencia de ingresos. Kang (2015, p. 499) habla directamente de "fuerte ethos igualitario"9 de los coreanos.

Sin embargo, si nos enfocamos en las clases medias altas y altas, ${ }^{10}$ sucede algo importante: alrededor de $20 \%$ no está de acuerdo ( $81 \%$ a favor) con que la desigualdad sea muy grande. La mayoría redistributiva se mitiga al dirigirnos a las clases medias altas y altas.

Si ampliamos la mirada a otras preguntas ${ }^{11}$ (Tabla 1 ), nuestra primera constatación se confirma: $28 \%$ de los encuestados de clase media alta y alta consideran justo que personas con más ingresos paguen por mejor atención a la salud (es decir, están a favor de la mercantilización de la atención a la salud) y un tercio entre ellos no considera que sea responsabilidad del Gobierno reducir las desigualdades (es decir, o identifican otras responsabilidades

9. Traducción propia, lo mismo que todas las traducciones de las páginas web, artículos de la prensa coreana y de investigadores, citados textualmente en este trabajo.

10. Es preciso indicar que se trata de autoidentificación de los encuestados, es decir quienes consideran ser parte de las clases media alta y alta. Unificamos éstas dos debido al pequeño número de respuestas de autoidentificación como clase alta, para acercarnos así hipotéticamente a quienes se consideran como parte de la élite socioeconómica. Somos conscientes del pequeño universo de lo que hemos llamado élite socioeconómica, de ahí sólo su utilidad de acercamiento descriptivo, que deberá ser complementado con otros universos más amplios.

11. Retomamos de Kang (2015) las preguntas de la tabla 1, aunque nosotros no sólo indagamos las respuestas en general sino que añadimos a las clases medias altas y altas. 
del Gobierno como prioritarias o no consideran como relevantes, en cuanto responsabilidad gubernamental, las políticas sociales redistributivas). Así, la mayoría redistributiva se mitiga al dirigirnos hacia la que identificamos como élite socioeconómica.

\section{Tabla 1}

Encuesta ISSP Corea 2009

\begin{tabular}{|c|c|c|c|}
\hline & $\begin{array}{l}\text { Diferencias de } \\
\text { ingreso son muy } \\
\text { grandes* }(1,589)\end{array}$ & $\begin{array}{l}\text { Es justo que } \\
\text { personas con } \\
\text { mayores ingresos } \\
\text { puedan pagar por } \\
\text { mejor cuidado en la } \\
\text { salud** }^{*}(1,598)\end{array}$ & $\begin{array}{l}\text { Responsabilidad del } \\
\text { Gobierno reducir } \\
\text { desigualdades*** } \\
(1,598)\end{array}$ \\
\hline $\begin{array}{l}\text { Totalmente de } \\
\text { acuerdo y de acuerdo }\end{array}$ & $90 \%(1,433)$ & $23 \%$ (368) & $75 \%(1,193)$ \\
\hline $\begin{array}{l}\text { Clases media alta y } \\
\text { alta }\end{array}$ & $81 \%$ (135) & $28 \%(46)$ & 67\% (112) \\
\hline \multicolumn{4}{|c|}{$\begin{array}{l}\text { * Pregunta: “¿Las diferencias de ingresos en Corea son demasiado grandes?” } \\
\text { ** Pregunta: “¿Es justo o injusto que las personas con mayores ingresos puedan comprar } \\
\text { una mejor atención médica?” } \\
\text { *** Pregunta: “¿Es responsabilidad del Gobierno reducir las diferencias de ingresos entre } \\
\text { las personas de altos ingresos y las de bajos ingresos?” } \\
\text { Entre paréntesis se indica el número de observaciones. }\end{array}$} \\
\hline
\end{tabular}

Fuente: elaboración propia con el apoyo técnico de Daniel Zazueta y de Yasodhara Silva a partir de la Encuesta ISSP Corea 2009 (The Social Science Research Institute, 2019).

Todavía más, si centramos la mirada en esta ocasión a preguntas binarias (entre dos valores), el consenso redistributivo se atenúa. La Encuesta de Valores 2010 (Inglehart et al., 2014) pregunta si el ingreso debe ser más igualitario (valor de la redistribución o igualitarismo) o si se requieren más diferencias en el ingreso de acuerdo con los esfuerzos individuales (valor del mérito individual o meritocracia): si centramos la atención sólo en los extremos (quienes están más de acuerdo o de acuerdo con una u otra de las opciones), sólo $30 \%$ están a favor de la igualdad y un fuerte $70 \%$ por el mérito individual; las clases medias altas y altas acentúan más la preferencia por el mérito individual, con $80 \%$ y sólo $20 \%$ con el igualitarismo (tabla 2 ). 
Tabla 2

Entre el mérito individual y la igualdad en Corea del Sur

\begin{tabular}{l|l|l}
\hline & $\begin{array}{l}\text { Preferencia por el mérito } \\
\text { individual* }\end{array}$ & $\begin{array}{l}\text { Preferencia por la igualdad de } \\
\text { ingresos* }\end{array}$ \\
\hline & Corea (2010) (1,190) & Corea (2010) (1,190) \\
\hline $\begin{array}{l}\text { Totalmente de acuerdo y } \\
\text { de acuerdo }\end{array}$ & $70 \%(432)$ & $30 \%(189)$ \\
\hline Clases media alta y alta & $80 \%(167)$ & $20 \%(42)$ \\
\hline
\end{tabular}

* Pregunta: “¿Qué tan de acuerdo está con la siguiente expresión: el ingreso debería ser más igualitario... Necesitamos mayores diferencias en el ingreso basado en los esfuerzos individuales?" Escala del 1 al 10, 1 más igualitario, 10 más esfuerzo individual. Se consideró de 1 a 3 Totalmente de acuerdo o de acuerdo con igualdad, del 8 al 10 Totalmente de acuerdo y de acuerdo con esfuerzos individuales.

Fuente: elaboración propia con el apoyo técnico de Daniel Zazueta y de Yasodhara Silva a partir de la Encuesta Mundial de Valores Corea del Sur 2010 (Inglehart et al., 2014).

No pretendemos explicar estas diferencias, para lo que se requerirían otras investigaciones; sólo deseamos concluir que el consenso redistributivo se atenúa y que entre las élites socioeconómicas (clases media alta y alta) al menos la tolerancia por la desigualdad es más importante que en el resto de las clases sociales (clases baja, trabajadora y media clase baja) y que porcentajes importantes de ellas prefieren la mercantilización de la salud y no le conceden importancia al rol redistributivo del Gobierno. Y que parece predominar la meritocracia frente a la igualdad. Nosotros ponemos aquí la atención en quienes no comparten un ethos redistributivo y sus preferencias en políticas sociales. Se trata de comprender si en las élites socioeconómicas (incluidas las empresariales) hay preferencia por la meritocracia o por políticas redistributivas.

Podrán comprenderse así las tendencias dominantes (enfoques) de los actores hegemónicos, las posibilidades de alianzas y acuerdos con otros actores sociales, y las resistencias a construir o reformar determinadas instituciones sociales. Es la siguiente parte de nuestra investigación: ¿cuáles son las preferencias de las organizaciones empresariales coreanas en relación con las políticas sociales en el periodo reciente (2009-2019)? ¿Estas preferencias se vinculan más fuertemente al ethos redistributivo o al ethos meritocrático? ¿Qué impacto tienen estas preferencias en la construcción de coaliciones de bienestar? 


\section{Organizaciones empresariales y bienestar en Corea del Sur: la Federation of Korean Industries y la Korea Enterprises Federation}

Para analizar las preferencias en políticas de bienestar de los sectores empresariales (dicho de otra manera, su enfoque ante los diversos caminos para enfrentar el problema de la desigualdad), una manera posible es seguir los posicionamientos de los principales organismos empresariales de Corea, completado con entrevistas con actores seleccionados. Para esta investigación se han analizado los posicionamientos (en sus páginas web y en declaraciones a la prensa) en torno a políticas sociales de dos organismos coreanos de particular interés: la Federation of Korean Industries (FKI) y la Korea Enterprises Federation (KEF) ${ }^{12}$ en el periodo de 2009 a 2019. Además, se complementó este estudio con una entrevista a funcionarios de la KEF. ${ }^{13}$

La FKI históricamente representó desde su fundación los intereses de los grandes conglomerados (hasta su crisis en 2016, en el contexto del escándalo Choi Soon-sil) ${ }^{14}$ y por ello ha tenido una membresía limitada en número, pero relevante en cuanto al poder de mercado; ${ }^{15}$ la KEF fue creada por la misma FKI en 1970 para promover su visión en torno a las relaciones laborales (Jun et al., 2018 , p. 66) y con actividades estrechamente vinculadas a la seguridad social. La primera, que ha disminuido su influencia y ha perdido a importantes socios desde 2016, se convierte de una institución lobby de los conglomerados más grandes, en un think tank con alcances más limitados en los últimos años; la segunda, que focalizó su atención en los asuntos laborales en su fundación y adquirió mayor importancia ante las fuertes movilizaciones sindicales de 1987, ha desempeñado un papel relevante en dar forma a las relaciones laborales coreanas como "asociación voluntaria que representa los intereses de clase de los (grandes) empresarios" (Jun \& Sheldon, 2006, pp. 211 y 219)

12. Mantenemos los nombres de estas organizaciones en inglés. Un trabajo más amplio debería incorporar más organizaciones empresariales y otras fuentes de información. Consideramos, sin embargo, que con estas limitaciones reconocidas es válido y pertinente el acercamiento propuesto aquí debido a la importancia de las dos organizaciones, en especial la FKI, en la historia empresarial y de las relaciones Estado-empresarios en Corea (Jun \& Sheldon, 2006; Jun et al., 2018).

13. Celebrada el 9 de julio de 2019 en Seúl, en las oficinas de esta organización.

14. Acusada de confabularse con la presidenta Park Geun-hye para extorsionar a empresarios. Este suceso llevó a la destitución de la presidenta y a su encarcelamiento. Choi Soon-sil también fue encarcelada y juzgada. Este escándalo estalló en 2016.

15. De acuerdo con la misma FKI (2020) en 2005 tenía 432 miembros, la mayor parte (366) empresas, en especial grandes conglomerados. 
y después con las comisiones tripartitas que iniciaron en el gobierno de Kim Dae-jung (Jun et al., 2018. pp. 75 y 79). La KEF recientemente, en el contexto de la reorganización de las instituciones empresariales ante la crisis de la FKI, amplió sus objetivos hacia una organización con perspectivas más generales (Korea Enterprises Federation [KEF], 2020a). ${ }^{16}$

En los objetivos de las dos instituciones, vinculados a los temas que interesan a este texto, se encuentran dos aspectos centrales: promoción de libre mercado y competitividad por una parte; promoción del bienestar y de políticas sociales asociadas al libre mercado, por otra. Coinciden fundamentalmente con el enfoque de la coalición pro-crecimiento (Valencia, 2019).

La FKI se veía como una institución creada para reforzar el libre mercado: "Éste es el principio fundamental y guía de la FKI y constituye el corazón de la identidad de la Federación y su razón de ser" (FKI, 2003). En 2003 la FKI señalaba como su Visión: "Fomentar una economía de libre mercado y dar el ejemplo de una empresa comercial confiable que sea completamente competitiva en el mercado global", e incluía en su Misión la "Activa contribución a la producción de bienestar y estabilidad en el empleo" (FKI, 2003). Poco antes, en 1999, después de la severa crisis de 1997-98 y del acceso a la Presidencia de Kim Dae-jung, la FKI publicaba su Carta de ética empresarial y señalaba: "Nuestro objetivo y misión es establecer un Estado de bienestar avanzado a través del crecimiento sostenido y el progreso, lo que nos permite transmitir una sociedad pacífica y rica a las generaciones que siguen" (FKI, 1999).

La KEF fue fundada en julio de 1970 y se dirigió desde el inicio a las actividades empresariales vinculadas con las relaciones laborales y después de la crisis de 1987 ha defendido posturas de flexibilización laboral (Jun et al., 2018, p. 73). El presidente Sohn Kyung-shik en 2019 describió de la siguiente manera la misión de la organización: "La Federación de Empleadores de Corea (KEF), establecida en 1970 con la misión de lograr una relación cooperativa entre la administración y el trabajo, se ha dedicado a construir la paz industrial y mejorar la competitividad nacional" (KEF, 2020a). Cuenta con servicios centrados en información y sugerencias sobre las políticas gubernamentales en empleo y seguridad social, en seguridad y salud, además de responsabilidad social de las empresas (KEF, 2020c). En julio de 2018 amplió sus objetivos para "'desarrollar políticas económicas y sociales basadas en la economía de

16. De tal manera que en 2020 representaba ya a 4,317 compañías, no sólo de los conglomerados, como en sus orígenes, sino también a empresas con menos de 300 trabajadores (Lee, 2020). 
libre mercado' y 'contribuir al desarrollo de una economía nacional sólida' (...) reforzando la competitividad empresarial” (KEF, 2018e).

Es destacable que ambas organizaciones se decantan por promover ya sea un "Estado de bienestar avanzado" o "políticas sociales" con vínculos al crecimiento y al libre mercado, en consonancia con la histórica coalición pro-crecimiento de Corea (Valencia, 2019). No rechazan per se al Estado de bienestar o a las políticas sociales sino que los vinculan dependientes del libre mercado, la competitividad empresarial y el crecimiento económico. No se observa a partir de los principios básicos de estas organizaciones una posición binaria: o bienestar o libre mercado, sino de bienestar a través del libre mercado. Veremos cómo se concretan estos objetivos en la promoción de políticas sociales y económicas en los años 2009 a 2019, sintetizada en la tabla 3 y detallada posteriormente. ${ }^{17}$ Para organizar los pronunciamientos en este tabla se utilizan los conceptos de salida, voz y lealtad de Hirschman (1977) ${ }^{18}$ La interrelación entre estos tres conceptos permite analizar una relación compleja entre participación, protesta y abandono, más allá de simples posiciones binarias (a favor y en contra).

Cabe recalcar que el periodo de estudio incluye las presidencias de Lee Myung-bak (2008-2012), Park Geun-hye (2013-marzo 2017), Moon Jae-in (mayo 2017-), las dos primeras consideradas de corte pro-empresarial, la tercera de tipo centro-izquierda. Dos años del periodo de Park Geun-hye (2016 y parte de 2017) se vieron envueltos en conflictos políticos vinculados a la corrupción y tráfico de influencias (caso Choi Soon-sil), con empresarios

17. Este trabajo, que se basa en posicionamientos de las federaciones elegidas, debe ser completado con el estudio de prácticas específicas en el marco de relaciones especialmente conflictivas entre conglomerados y organizaciones de trabajadores. Históricamente las grandes empresas coreanas han sido particularmente reacias a las organizaciones sindicales, si no es que en algunos casos, como Samsung, han sido claramente anti-sindicales (Valencia, 1996; Shin, 2003).

18. Originalmente estos conceptos fueron desarrollados por Hirschman especialmente para analizar la relación entre empresas e individuos (consumidores), pero han sido utilizados en diversos análisis que buscan desentrañar la relación entre organismos o políticas y participantes. Aquí lo aplicamos de una manera simplificada a la relación entre políticas sociales y organizaciones empresariales. Por lealtad, lo que Hirschman (1977, p. 79) llama "apego especial a un organismo", aquí entenderemos apego y participación en una política social que puede expresarse en cumplimiento de reglas u opiniones favorables; por voz crítica la expresión de "insatisfacción" o "protesta general" (Hirschman, 1977, p. 14) que puede expresarse en críticas a las políticas sociales o a las propuestas de modificación; por salida, el abandono del organismo (Hirschman, 1977, p. 14) o aquí una forma de abandono de las políticas sociales vigentes; la opción de la salida en nuestro caso también puede implicar en primer lugar una amenaza de salida. 
mezclados, ${ }^{19}$ lo que impactó no sólo en la destitución de la presidenta Park sino también en el encarcelamiento de Lee Jae-yong, vicepresidente de Samsung Electronics y futuro heredero del conglomerado. Los grandes conglomerados resultaron también afectados ante la imagen pública de colusión entre políticos, gobernantes y empresarios.

Veamos en los próximos apartados el detalle de esta tensión entre "lealtad" y "salida", con la presencia de la "voz crítica" de organizaciones empresariales en diversas coyunturas, en temáticas vinculadas a las políticas sociales: seguimos los siete apartados de la tabla síntesis siguiente, dos sobre cuestiones generales como la economía política y la protección laboral (vinculadas estrechamente a las políticas sociales) y cinco sobre políticas sociales específicas como la seguridad social en general, los seguros de salud, pensionarios y contra accidentes de trabajo, y respecto de las políticas de cuidado. ${ }^{20}$

\section{Tabla 3}

Organizaciones empresariales y políticas sociales en Corea del Sur (2009-2019)

\begin{tabular}{|c|c|c|c|}
\hline Institución & $\begin{array}{l}\text { Lealtad } \\
\text { (Participación) }\end{array}$ & $\begin{array}{l}\text { Voz crítica } \\
\text { (Participación) }\end{array}$ & $\begin{array}{l}\text { Salida o amenaza } \\
\text { de salida }\end{array}$ \\
\hline $\begin{array}{l}\text { Economía } \\
\text { política }\end{array}$ & $\begin{array}{l}\text { En favor del } \\
\text { diálogo tripartita. }\end{array}$ & $\begin{array}{l}\text { Exceso de regulaciones } \\
\text { gubernamentales. }\end{array}$ & $\begin{array}{l}\text { Resistencia a } \\
\text { la inversión } \\
\text { productiva. }\end{array}$ \\
\hline $\begin{array}{l}\text { Protección } \\
\text { laboral }\end{array}$ & $\begin{array}{l}\text { Contribución } \\
\text { reglamentada. }\end{array}$ & $\begin{array}{l}\text { Exigencia de mayor flexibilidad } \\
\text { de relaciones laborales (frente } \\
\text { a "excesiva" protección laboral), } \\
\text { de la contratación temporal y } \\
\text { de menor jornada laboral con } \\
\text { reducción salarial. }\end{array}$ & $\begin{array}{l}\text { Relocalizar } \\
\text { manufactura } \\
\text { en mercados } \\
\text { emergentes por } \\
\text { altos costos } \\
\text { laborales. } \\
\end{array}$ \\
\hline
\end{tabular}

19. Puede verse en Choi (2016) el rol adjudicado a la FKI en este conflicto.

20. También estuvieron presentes posicionamientos empresariales en torno a las comidas escolares gratuitas y a la filantropía. Tuvieron presencia menor en las fuentes de información consultadas. Decidimos no incorporarlas en este trabajo. 
Enrique Valencia Lomelí

\begin{tabular}{|c|c|c|c|}
\hline $\begin{array}{l}\text { Seguros } \\
\text { contributivos en } \\
\text { general }\end{array}$ & $\begin{array}{l}\text { Reconocimiento } \\
\text { a la generación de } \\
\text { seguridad social } \\
\text { en la historia } \\
\text { económica } \\
\text { coreana. } \\
\text { Contribución } \\
\text { reglamentada. }\end{array}$ & $\begin{array}{l}\text { Incrementos de costos laborales } \\
\text { mina la competitividad } \\
\text { productiva. }\end{array}$ & $\begin{array}{l}\text { Crecimiento } \\
\text { de la inversión } \\
\text { coreana en el } \\
\text { exterior por } \\
\text { exceso de cargas } \\
\text { sociales o altos } \\
\text { costos para } \\
\text { empresas. } \\
\text { Resistencia a, o } \\
\text { reducción de la } \\
\text { inversión. } \\
\text { Mayor } \\
\text { automatización } \\
\text { en los procesos } \\
\text { productivos } \\
\text { (menor } \\
\text { contribución). } \\
\end{array}$ \\
\hline $\begin{array}{l}\text { Pensiones: } \\
\text { extensión de la } \\
\text { edad de retiro } \\
\text { ( } 55 \text { a } 60 \text { años) } \\
\text { (Ley Laboral, } \\
\text { abril 2014) }\end{array}$ & $\begin{array}{l}\text { Extensión edad de } \\
\text { retiro en algunos } \\
\text { conglomerados. } \\
\text { Incorporación } \\
\text { del "sistema de } \\
\text { tope salarial" en } \\
\text { conglomerados. } \\
\end{array}$ & $\begin{array}{l}\text { Exigencia de adoptar el "sistema } \\
\text { de tope salarial" ante extensión } \\
\text { de edad de retiro para evitar } \\
\text { incremento de costo laboral y } \\
\text { para impulsar responsabilidad } \\
\text { intergeneracional. }\end{array}$ & \\
\hline $\begin{array}{l}\text { Sistema de } \\
\text { Salud }\end{array}$ & $\begin{array}{l}\text { Reconocimiento } \\
\text { a la cobertura } \\
\text { prácticamente } \\
\text { universal del } \\
\text { seguro de salud. }\end{array}$ & $\begin{array}{l}\text { Deben eliminarse regulaciones } \\
\text { que impiden la inversión privada } \\
\text { en instituciones médicas y no } \\
\text { incrementarse las cargas del } \\
\text { seguro de salud. }\end{array}$ & \\
\hline $\begin{array}{l}\text { Seguro ante } \\
\text { accidentes de } \\
\text { trabajo }\end{array}$ & $\begin{array}{l}\text { Apoyo a la } \\
\text { seguridad en el } \\
\text { trabajo. }\end{array}$ & $\begin{array}{l}\text { Crítica ante las nuevas } \\
\text { medidas para seguridad en el } \\
\text { trabajo (prohibición de uso de } \\
\text { trabajadores subcontratados en } \\
\text { empleo peligrosos). } \\
\text { Crítica a nueva Ley sobre } \\
\text { "Accidentes de Trayecto", a } \\
\text { excesivo castigo por muerte } \\
\text { accidental de trabajadores y a la } \\
\text { posible obligación de divulgar } \\
\text { datos sobre seguridad y salud en } \\
\text { las empresas. }\end{array}$ & \\
\hline
\end{tabular}




\begin{tabular}{l|l|l|l}
\hline Políticas de & Apoyo al gasto & Exigencia de mayor flexibilidad & \\
cuidado infantil & público. & de relaciones laborales. \\
Creación de & Ventajas adicionales para & \\
instituciones & el cuidado infantil genera \\
empresariales de & mayor déficit en el seguro de \\
cuidado infantil & desempleo. & \\
para trabajadores. & $\begin{array}{l}\text { Urgencia de personalizar } \\
\text { sistemas de cuidado infantil. }\end{array}$ & \\
\hline
\end{tabular}

Fuente: elaboración propia a partir de posicionamientos de organismos empresariales en páginas web, en entrevistas a medios de comunicación y en entrevista realizada por el autor de este texto. Pueden verse los detalles de estas múltiples fuentes en los siete apartados descritos posteriormente.

\section{Organizaciones empresariales y bienestar en Corea del Sur: economía política y protección laboral}

En primer lugar, respecto a los debates generales de economía política vinculados a las políticas sociales, Sohn Kyung-shik, nuevo presidente de KEF en 2018, se pronunció a favor del diálogo tripartita a fin de "mejorar nuestros fundamentos económicos y sociales para generar más empleos para nuestra generación futura" y para enfrentar la cuarta revolución industrial (KEF, 2018b). Sin embargo, la KEF (2018a) cuestiona las excesivas regulaciones en el mercado laboral que deben ser reformadas hacia la flexibilidad; las leyes laborales rígidas y uniformes basadas en "los trabajos tradicionales de manufacturación fabril no pueden regular muchas formas de empleo o relaciones laborales que se están volviendo cada vez más variables" (KEF, 2018a). La KEF es insistente: "Sin una reforma regulatoria, no habrá creación de empleo en el futuro", en el marco de la competencia con China; la propuesta de "crecimiento dirigido por el ingreso y crecimiento innovador" (estrategia propuesta por el presidente Moon Jae-in) no debe discriminar el "crecimiento por la inversión" (KEF, 2018a).

Estas regulaciones tienen su efecto de "salida": un ejecutivo de FKI declaraba en 2012 que el decrecimiento en la inversión es causado por la excesiva intervención gubernamental; según The Korea Exchange los 10 principales conglomerados han aumentado notablemente su "ratio de ganancias retenidas" (de 600 a 1,219\% entre 2004 y 2011) (Chung, 2012). En la misma línea, en 2015 un ejecutivo de FKI aceptaba que "Un incremento de salario y más gasto en programas de bienestar son necesarios", pero el estímulo al creci- 
miento económico debe de incluir el corte de regulaciones gubernamentales y el impulso a la economía creativa (Kim, 2015).

En segundo lugar, las organizaciones empresariales postulan claramente, de diversas maneras, su preferencia por la flexibilización de las relaciones laborales y la reducción de cargas laborales que permitiría mayor productividad. Ciertamente, las empresas por una parte expresan su aceptación para contribuir a la protección de los trabajadores, pero por otra cuestionan con su voz crítica lo que consideran excesivas protecciones. Desde 2009, tres organizaciones - FKI, KEF y la Korea Federation of Small and Medium Bussines (K-Biz) - se opusieron al límite de contratación de dos años de los trabajadores temporales: "el límite en el periodo de contratación de trabajadores eventuales debe ser abolido" (Kim, 2009).

Incluso, ante la medida anunciada por parte del Gobierno en 2014 para reforzar la cobertura de la seguridad social a los trabajadores de tiempo parcial (con empleo de menos de 60 horas al mes), la KEF (2014c) manifestó que crearía severas cargas administrativas a las empresas y que podría provocar bajas en los ingresos de estos trabajadores; se trata de una medida que reduce la flexibilidad del trabajo y que afecta negativamente la creación de empleos. Poco antes de esta declaración, la KEF (2013c) había publicado un estudio comparativo con seis países que alcanzaron la tasa de $70 \%$ del empleo en relación con la población y su conclusión fue que es mejor establecer un mercado laboral flexible, que excesiva protección laboral, con "varios tipos de empleo, como puestos a tiempo parcial y temporales, y más empleo para grupos vulnerables, incluidas las mujeres"; los países comparados fueron Alemania, Holanda, Gran Bretaña, Estados Unidos, Nueva Zelandia y Canadá.

En 2015 las organizaciones empresariales acentuaron sus presiones por una reforma laboral más flexible. En ese año se presentó una declaración conjunta de FKI, KEF, K-Biz y otras organizaciones empresariales en favor de la flexibilidad laboral; para el vicepresidente de KEF, Kim Young-bae, los trabajadores mayores que están listos para el retiro obtienen hasta tres veces más salario que los nuevos empleados, por lo que debe eliminarse el "sistema basado en la antigüedad" (senior based-system) por el de "salario centrado en el desempeño" (performance-centered salary); estas organizaciones demandan una reforma laboral que permita mayor facilidad en los despidos (Lee, 2015b); la considerada excesiva protección laboral "con derechos establecidos" lleva a desempleo de jóvenes y de trabajadores no regulares (KEF, 2015d). El presidente de la KEF, Kim Young-vae, destaca que la necesidad de distender o 
disminuir la protección laboral no significa "agravar la inseguridad del empleo" sino proveer más empleo para más personas (KEF, 2015a).

Poco después, en 2016, el mismo funcionario de la KEF (2016a) señaló que los "proyectos de ley de reforma laboral son sólo el primer paso en nuestros esfuerzos hacia un mercado laboral flexible y justo"; se refería a los acuerdos alcanzados por la Comisión Tripartita en septiembre de 2015 y que estaban pendientes en la Asamblea Nacional; y la FKI sugirió reducir la jornada laboral: "jornadas más cortas beneficiarían tanto a los empleadores como a los empleados si los trabajadores aceptan una disminución salarial y la administración contrata a más trabajadores", para que las empresas puedan contratar más jóvenes y ampliar el empleo para trabajadores veteranos. Señala que la reducción de jornada laboral ya es aplicada en algunas empresas de manera exitosa, lo que permite reducir costos laborales (Yoon, 2016).

Está presente así el planteamiento de un pacto intergeneracional en el que teóricamente los jóvenes resultarían beneficiados (con empleos y mejores salarios), a diferencia de los adultos mayores, que deberían sacrificar sus beneficios y derechos presentes, pacto con raíces históricas en las propuestas de la coalición pro-crecimiento. ${ }^{21}$

De nuevo, las tensiones entre lealtad (contribución a la protección laboral) y voz crítica (excesivas protecciones) conducen hacia caminos de "salida". Después de que la Corte Suprema dictaminara en diciembre de 2013 que los bonos pagados regularmente deberían incluirse como salarios ordinarios, dijo un funcionario de la FKI: "Tiene sentido que las empresas relocalicen sus bases manufactureras clave en los mercados emergentes que tienen un atractivo claro en los costos laborales" (Kim Y., 2013). Critican que con esa medida sobre los bonos podrían aumentar los costos de las pensiones. Por eso, de acuerdo con Y. Kim (2013), varias compañías del sector electrónico (por ejemplo, LG y Samsung) han deslocalizado ya parte de su producción y un funcionario de la FKI manifestó "que no descartaba la posibilidad de que los fabricantes de automóviles que operan en Corea salgan debido al endurecimiento del mercado laboral". Se puede resumir la tensión de esta manera: el enfoque centrado en las protecciones sociales genera costos en las empresas; el enfoque centrado

21. Para esta coalición, el bienestar de los hogares y los trabajadores en los años de fuerte crecimiento coreano (desde los años sesenta) es secundario; lo central es el crecimiento económico, el bienestar sería para las generaciones futuras gracias al trabajo de las generaciones de trabajadores en activo. Hay un intercambio: o crecimiento o bienestar actual. De este espíritu surgieron las propuestas históricas de políticas sociales mínimas (véase Valencia, 2019). 
en la flexibilidad laboral asegura hipotéticamente menores costos, mayor productividad y empleo, en línea con la histórica coalición pro-crecimiento.

\section{Organizaciones empresariales y bienestar en Corea del Sur: las políticas sociales}

Las organizaciones empresariales estudiadas se van posicionando también en relación con las propuestas de reformas a diversas políticas sociales. Veamos de nuevo la interrelación entre lealtad, voz y salida en estas pugnas sobre cambios a las políticas sociales en particular (seguros sociales en general, sistema de pensiones, seguro de salud, seguro contra accidentes de trabajo y políticas de cuidado).

En primer lugar, la KEF evalúa positivamente la trayectoria histórica de los seguros sociales:

(...) al proporcionar diversos dispositivos institucionales basados en la Ley Orgánica de Seguridad Social, hemos podido mejorar la calidad de vida al proteger la ciudadanía de riesgos sociales como el parto, la crianza de los hijos, el desempleo, la vejez, la discapacidad, la enfermedad, la pobreza y la muerte; [este] sistema de seguridad social ${ }^{22}$ se ha desarrollado en un pequeño periodo de 31 años, con "alta incorporación" (...) Lo que en Europa llevó 100 años, en Corea del Sur 31 (KEF, comunicación personal, 9 de julio 2019).

A esta trayectoria, aduce, las empresas han aportado y la insistencia empresarial expresada en informes periódicos ${ }^{23}$ es su "lealtad" en la contribución creciente de las compañías, incluso en comparaciones internacionales. El reporte 2014 "Estado actual de la carga del seguro social y sugerencias de mejora" señalaba que el aporte contributivo de los empleadores representa el $45.2 \%$ de un total de 88,796 millones de dólares de los cinco principales seguros (seguro de salud, pensión nacional, seguro de desempleo y de accidentes industriales, además del seguro de cuidado de larga duración) (KEF, 2014b)..$^{24}$ El reporte de 2016 apuntaba en $45.4 \%$ el aporte contributivo de

22. Se refiere la KEF al seguro de accidentes de trabajo, de salud, de pensiones, de desempleo y al de cuidado de largo plazo. También a los programas de asistencia social frente a la pobreza.

23. La KEF insiste en reportes anuales en la participación concreta de las empresas en la seguridad social con contribuciones crecientes. Citaremos más adelante varios de ellos.

24. Véase reporte de 2015 (KEF, 2015e). 
los empleadores, de un total de 97,650 millones de dólares; el costo social pagado por trabajadores y empleadores pasó de $16 \%$ en 2005 a $18.7 \%$ del salario total (KEF, 2016c). El reporte de 2017 concluyó que la contribución total para los cinco principales seguros se incrementó en $6.1 \%$; que la contribución entre 2007 y 2017 ha aumentado 7.9\% en promedio anual (frente a $5.2 \%$ del crecimiento del producto interno bruto [PIB] y 2.3\% de la inflación); y que el gasto en seguridad social nacional llegó a 6.9\% del PIB en 2016 (por abajo del promedio de la Organización para la Cooperación y el Desarrollo Económico [OCDE], 9.2\% del PIB, pero segundo entre los países no europeos de esta organización) (KEF, 2018j).

Este crecimiento, sin embargo, no puede ser sostenido y provoca la "voz crítica" de las organizaciones empresariales: "la capacidad de las empresas y las personas para llevar su carga contributiva llegó ahora al límite” (KEF, 2018j). Mientras la productividad de los trabajadores de los principales 30 conglomerados se ha reducido entre 2011 y 2015 , los costos laborales por empleado se han incrementado prácticamente en $18 \%$, de acuerdo con estudio de la FKI (Korea Times, 2016b). ${ }^{25}$ Los aumentos en los salarios mínimos de los últimos años (2018 y 2019) generaron airadas protestas: ante el aumento de $16.4 \%$ en el salario mínimo anunciado para 2018 (fijado a 7,530 wons la hora), KEF (2017c) manifestó fuerte preocupación porque resultará en "enorme costo laboral adicional" en las micro y pequeñas empresas, y dificultará la creación de empleos; de nuevo se opuso ante el aumento anunciado para 2019 de 10.9\% (salario por hora fijado en 8,350 wons) porque incrementará la carga de los costos laborales para las pequeñas y medianas empresas; es inaceptable de acuerdo con KEF (2018f) que el aumento refleja que " 4.9 puntos porcentuales apuntan a mejorar la distribución actual del ingreso".

Son cuestionables la "baja satisfacción de los beneficiarios" a pesar del incremento de presupuestos y "el estancamiento en la ceguera del bienestar" con cargas para las empresas muy altas y con tendencia a "centrarse en la cobertura de los seguros" (KEF, comunicación personal, 9 de julio de 2019). Así, es recurrente la "voz crítica" que enfatiza los fuertes costos laborales para las empresas producto de estos seguros. Críticas severas como las mencionadas conducen por una parte a propuestas $y$, por otra, nuevamente a tensiones hacia la "salida": en cuanto a las primeras, KEF (2018h) presentó propuestas de modificación a ocho leyes porque considera que se ha deteriorado la atmós-

25. Véase también KEF (2009, 2014b). 
fera empresarial por la intensificación de las cargas a las compañías, "junto con el aumento del salario mínimo y el salario ordinario, la reducción de las horas de trabajo y el aumento de los costos de la seguridad social, de acuerdo con la continuidad del sistema económico 'alto costo y bajo rendimiento"'. En cuanto a las segundas, de acuerdo con un reporte de la FKI de 2013, un creciente número de empresas están deslocalizando su producción manufacturera al exterior a causa de fuertes regulaciones, conflictos laborales, altos costos y sentimientos antiempresariales (Kim, R., 2013). De acuerdo con el vicepresidente de la FKI en 2017, Kwon Tae-shin, mientras en Estados Unidos los impuestos corporativos bajan, en Corea algunos candidatos presidenciales proponen aumentarlos de 22 a $25 \%$; esa medida junto con el incremento en los costos laborales y las regulaciones gubernamentales puede llevar a empresas locales a invertir en el exterior (Lee, 2017).

De acuerdo con el reporte sobre el incremento de los costos de seguridad social (seguro de salud, pensión nacional, seguro de desempleo y de accidentes industriales) elaborado por la KEF en 2009, éstos han aumentado en 4.1 veces entre 1997 y 2008, frente al crecimiento de 2.8 veces del PIB, el segundo aumento más fuerte de acuerdo con la OCDE; el que más ha aumentado es el seguro de salud: 6.9 veces y el peso del gasto de seguridad social para las empresas se ha cuadruplicado; según esta organización, el gasto excesivo en seguridad social disminuye la motivación para invertir y causa inestabilidad en el empleo (KEF, 2009).

Entre otras medidas, propone la KEF fijar objetivos de protección social y asegurar la división de roles entre Gobierno y empresas, frente al bajo gasto gubernamental (KEF, 2009). El reporte de 2014, que añade a los cuatro seguros mencionados, el seguro de cuidado de largo plazo, señala que de acuerdo con la OCDE los gastos de seguridad social han aumentado de $4.7 \%$ en 2003 a $6.6 \%$ en 2012 y que la tasa de crecimiento ha sido de $39.6 \%, 10$ veces el promedio de los países de la OCDE (4\%); además indica que los gastos que más han aumentado son los del seguro de salud y de pensiones. Si se mantiene el sistema actual, el gasto en seguridad social aumentará a 23.2\% del PIB en 2060. Estos incrementos afectan negativamente al empleo y a la inversión. Por todo ello, la KEF (2014b) propone varias medidas: por ejemplo, fijar niveles apropiados de seguridad social y límites razonables a esta carga, además de mejoras en los seguros.

El reporte de 2015 concluye en que se requieren medidas urgentes para la "sostenibilidad de los sistemas de seguridad social" (KEF, 2015e). El 17 de 
diciembre de 2018, 17 organizaciones empresariales (en primer lugar KEF, además de Korea International Trade Association, KITA, y K-Biz) mostraron su inconformidad ante la pretensión del Gobierno de cambiar el "Decreto de cumplimiento del salario mínimo" para incluir las horas de descanso semanal y otras horas incluidas en los contratos colectivos como horas trabajadas y sometidas al cálculo del pago del salario mínimo; esta medida incrementaría el costo laboral de las empresas y como resultado "las empresas coreanas están perdiendo su competitividad global y su motivación económica para invertir y emplear" (KEF, 2018i). Funcionarios de la KEF sintetizaron esta problemática de la siguiente manera:

Es una realidad que el aumento de la carga sobre los costos de bienestar social, junto con el empeoramiento de las condiciones comerciales debido a la reciente incertidumbre económica y social, está dificultando los incentivos para atraer las inversiones futuras de las empresas y la capacidad de dichas inversiones (KEF, comunicación personal, 9 de julio de 2019).

En segundo lugar, respecto en particular al caso de las pensiones, destacan los posicionamientos empresariales de reconocimiento y voz crítica. El reconocimiento por la generación de seguro a partir de 1988 que "actualmente es el tercer fondo más grande del mundo" que beneficia a 21.92 millones de personas (a marzo de 2019) y que garantiza los ingresos de adultos mayores en Corea (KEF, comunicación personal, 9 de julio de 2019). La voz crítica se dirigió en el periodo de estudio a la extensión de la edad de retiro, de 55 a 60 años en la Ley Laboral de abril de 2014, inicialmente aplicable a los empleados en empresas con más de 300 trabajadores (Choi, 2014).

Los organismos empresariales KEF, FKI, K-Biz, Korea Chamber of Commerce and Industry — KCCI- y KITA publicaron en abril de 2013 un comunicado crítico conjunto: "La extensión obligatoria de la edad de jubilación y las vacaciones públicas obligatorias sin precedentes (...) causan una cantidad astronómica de costos laborales y disputas en las relaciones laborales" (KEF, 2013b). En estudio de la KEF más de la mitad de las compañías coreanas están preocupadas por los impactos del incremento obligatorio de la edad de 
retiro; muchas de estas compañías prefieren el "sistema de tope salarial"26 (KEF, 2013a). El secretario general de FKI, Im Sang-hyung ante la decisión de Samsung de extender la edad de retiro de 55 a 60 años y cortar el salario de los trabajadores en $10 \%$ por cada año adicional trabajado, señaló: "A menos que las empresas adopten el 'sistema de tope salarial' a cambio de una edad de jubilación extendida para sus trabajadores, los costos laborales aumentarán y pesarán en sus resultados" (Choi, 2014). La introducción del "sistema de tope salarial" ha ido avanzando: de acuerdo con un estudio de KEF en 2015 (2015f), 51.4\% de las 200 empresas más grandes lo habían introducido; y en un estudio de 2017, 52.2\% de las 300 empresas más grandes ya lo habían hecho (KEF, 2017a).

La voz crítica de algunas organizaciones empresariales propone sacrificios en salarios y programas sociales a la generación de trabajadores de los años cincuenta y principios de los sesenta. El vicepresidente de FKI, Lee Seung-cheol, señaló en 2015 que "Los baby boomers, los nacidos entre 1955 y 1963, deberían hacer sacrificios para mejorar las cosas para las personas de la próxima generación compartiendo trabajos bien remunerados con ellos y evitando que las pensiones y otros programas de asistencia social sean demasiado generosos para la generación pasada", y compartir beneficios con generaciones jóvenes nacidas entre 1979 y 1992 "que han estado luchando por encontrar trabajo y acumular riqueza en medio de la desaceleración económica de los últimos años". Añadió: "Es triste descubrir que la mayoría de los jóvenes no esperan recibir una pensión cuando se jubilen, ya que el Fondo Nacional de Pensiones se agotará en 2060" (Lee, 2015a).

Proponen así estas organizaciones empresariales un difícil intercambio: reducción salarial y pensionaria por empleo de los adultos mayores de 55 años, y más empleos con mejores salarios para las generaciones jóvenes, para asegurar menores costos laborales de las empresas y mayor productividad;

26. La KEF (2020b) define de la siguiente manera este sistema: "El sistema de tope salarial se refiere a la reducción gradual de los salarios de los trabajadores adultos mayores a cambio de que continúen en el empleo después de que alcancen cierta edad. Si bien hasta el momento no ha habido una definición académica clara del sistema de tope salarial, generalmente se considera que es una herramienta para reducir los salarios de los trabajadores mayores en consonancia con su nivel reducido de productividad, mientras que al mismo tiempo les brinda seguridad laboral". Esto significa que los trabajadores mayores de 55 años estén dispuestos a laborar con una reducción porcentual anual acumulativa de su salario hasta llegar a la edad de retiro de 60 años. 
plantean así un duro acuerdo intergeneracional cercano a la trayectoria de la coalición pro-crecimiento.

En tercer lugar, ahora respecto al caso específico del seguro de salud, de nuevo aparece la tensión entre reconocimiento y voz crítica. En entrevista, funcionarios de la KEF reconocían lo siguiente:

Después de introducir el sistema de seguro médico en 1977 por primera vez, se expandió a la población nacional en 1989 después de 12 años. El registro más corto sin precedentes en la historia del seguro social de salud en el mundo, que contribuyó a la mejora de los estándares nacionales de salud a medida que toda la población recibe beneficios del seguro de salud (KEF, comunicación personal, 9 de julio de 2019). ${ }^{27}$

Sin embargo, la voz crítica subraya de nuevo los incrementos en los costos. La KEF (2015c) anunció de diversas maneras su oposición al incremento de "costos": primero de la prima del seguro de salud en 2015 en $0.5 \%$ en el gobierno de Park porque lo que se requiere es mejorar la eficiencia en el gasto del seguro de salud; y segundo en 2018, ya en el gobierno de Moon, de la prima del seguro de cuidado de largo plazo de 7.38 a $8.51 \%$ incluida en la prima del seguro personal de salud, porque a fin de cuentas:

Aunque el seguro de cuidado a largo plazo parece haber aumentado la carga tanto para los trabajadores como para las empresas por el mismo margen, es probable que las compañías asuman la mayor parte de los costos adicionales porque es posible que la carga de los trabajadores se compense mediante aumentos salariales (KEF, 2018g).

Además, ante el anuncio en 2019 del plan de Moon Jae-in para reforzar la cobertura del seguro nacional de salud ("más contribuciones para mayor cobertura para 2019-2023”), que apuntala el plan 2017-2022 que cubre todos los servicios médicos excepto los cosméticos, KEF (2019) señala que las empresas "no tienen capacidades suficientes para soportar una carga de costos adicional" y propone al Gobierno que "mejore la implementación de la política [de salud] de manera que minimice la carga nacional, teniendo en cuenta que

27. Incluso en esta entrevista uno de los funcionarios señaló que "orgullosamente" 97\% de la población está en el seguro de salud, es como "una utopía": todas las personas están incluidas. 
el gasto en atención médica aumentará considerablemente a medida que el envejecimiento de la población cobre impulso".

Y por último, en referencia a las llamadas "reglas Galápagos" porque sólo existen en Corea, la FKI había propuesto en 2016 siete reformas de regulaciones existentes, entre ellas la prohibición de inversiones privadas en instituciones médicas; autorizarlas permitiría la creación de 269,000 empleos y nueva inversión cercana a 14,000 millones de dólares (Korea Times, 2016a).

En cuarto lugar, frente a las modificaciones propuestas al seguro ante accidentes de trabajo, la KEF (2017b) apoya de diversas maneras la necesidad de proteger a los trabajadores de los "accidentes de desplazamiento"; véase también KEF (2017d): "Está claro que todos los miembros de la sociedad, incluidas las empresas, siempre desean lograr lugares de trabajo seguros a través de la innovación en la seguridad laboral". Se trata de una voz cercana a la "lealtad" de Hirschman.

Sin embargo, lo que predomina son las narrativas (voz crítica) contra el exceso de responsabilidades que pretenden las autoridades cargar a las empresas y los efectos en la competitividad: por ejemplo, ante los "Planes integrales sobre innovación en seguridad y salud en el trabajo" anunciados por el Gobierno en febrero de 2015, KEF (2015b) sostiene que "los planes enfatizan excesivamente el deber y la responsabilidad de los contratistas primarios, imponen deberes conjuntos de seguridad y salud ocupacional, y prohíben el uso de trabajadores subcontratados para trabajos arriesgados/peligrosos", además de que este camino "conducirá a restringir la autonomía de las empresas en la administración de la mano de obra". El plan de acción en torno a esta obligación fue publicado el 3 de julio de 2017 y KEF (2017d) expresó su inconformidad. De acuerdo con la KEF (2017b) las responsabilidades de los accidentes de trabajo recaen también en los trabajadores, por ello "es excesivo compensar los accidentes industriales sin ninguna limitación, aun en caso de que los accidentes se deban a negligencia grave de los trabajadores". Incluso, poco después KEF (2018c) propuso cambios a la Industrial Safety Health Law, debido al excesivo castigo de al menos un año de prisión por muerte de un trabajador, porque "la ocurrencia de accidentes industriales es causada por 
una combinación de varios factores, como la conciencia insuficiente de seguridad de los empleados y la violación de las obligaciones de los empleadores". ${ }^{28}$

Por último, la KEF se posicionó en abril de 2018 ante la diversas decisiones judiciales y gubernamentales que favorecen la publicación de información sobre substancias peligrosas en el trabajo; si bien esta información es necesaria para identificar las enfermedades laborales, "es necesario considerar el efecto dominó en la seguridad o economía nacional, la participación en el mercado nacional e internacional de los productos y la brecha tecnológica entre países" en el contexto de una fuerte competencia en la industria de semiconductores y pantallas (el gap entre China y Corea se ha reducido sólo a dos o tres años), de acuerdo con KEF (2018d): "Esto suscita controversias sobre preocupaciones en torno a la divulgación excesiva de información confidencial y secretos comerciales".

Tanto en el caso de este seguro contra accidentes en el trabajo como en el seguro de salud, las organizaciones empresariales estudiadas manifiestan un reconocimiento explícito y en algún caso sus logros en contexto internacional; sin embargo, en la dinámica de defensa de los intereses empresariales levantan su voz crítica ante los llamados excesos de costos y de regulaciones. En la repartición de tareas de bienestar entre empresas, Estado y sindicatos, estas organizaciones empresariales pugnan por menos responsabilidades para ellas y proponen duros acuerdos intergeneracionales para reducir los costos laborales.

En quinto lugar, ante la problemática demográfica de Corea (creciente envejecimiento y baja tasa de fecundidad) y la necesidad de incrementar la participación laboral femenina, los empresarios manifestaron en este periodo de estudio, en diversos foros, su reconocimiento al problema de la "baja tasa de natalidad" y a la necesidad de acciones ante él. Su preocupación central es la reducción del dinamismo económico (crecimiento) en un contexto de baja participación femenina y "baja tasa de natalidad". Por ejemplo, en declaración conjunta de acciones de KEF, FKI, KITA, K-Biz y KCCI en favor de la superación de la baja tasa de natalidad, entendida como problemática no individual sino tarea nacional, proponen "Crear una atmósfera para equilibrar el cuidado in-

28. Se trata de un asunto sensible. Corea históricamente ha tenido una alta tasa de muertes por accidentes industriales (Valencia, 1996). De acuerdo con las estadísticas del Instituto Coreano del Trabajo (Korea Labor Institute, 2017), entre 2000 y 2015 hubo, en promedio anual, 2,366 muertes por estos accidentes. 
fantil y la vida laboral", expandir facilidades de cuidado infantil en las empresas y promover el permiso de paternidad "para facilitar las tareas domésticas y el cuidado de los niños para las madres" (KEF, 2015g). ${ }^{29}$

El problema al que estas organizaciones apuntan es a la reducción del crecimiento económico:

La OCDE advirtió que la tasa de crecimiento potencial de Corea en 2034 se reduciría a un rango del $1 \%$ si la baja tasa de participación de las mujeres en la fuerza laboral (57.9\%) y la tasa de natalidad (1.24 personas) no se recuperan al nivel de los países avanzados (OCDE 1.68 personas) (KEF, 2016b).

Sin embargo, la tensión se renueva entre la "lealtad" y la "voz", con repetidos planteamientos críticos de nuevo ante la resentida como creciente carga para las empresas: lo que se requiere es flexibilizar las relaciones laborales, es de nuevo el leitmotiv empresarial.

Ante planes gubernamentales dirigidos a la temática del cuidado, las organizaciones empresariales tomaron la palabra. De nuevo aparece la tensión entre reconocimiento (en este caso a una problemática y la necesidad de enfrentarla) y voz crítica por los excesivos costos laborales y en busca de la flexibilización laboral. Frente a la propuesta de plan de cinco años del gobierno de Lee Myung Bak "para impulsar la descendente tasa de natalidad, el empleo femenino y lidiar con el envejecimiento de la sociedad", el enfoque de la KEF (2010) fue que la ley coreana de protección del empleo para las mujeres no es inferior en comparación con la de los países avanzados; ${ }^{30}$ Corea mantiene un alto nivel de bienestar, como la licencia de paternidad para los

29. Véase también $\operatorname{KEF}(2010,2016 c)$.

30. Desde 2001, la Ley de Protección de la Maternidad amplió la licencia de maternidad de 60 días a 90 días, con $100 \%$ de reposición de salario en los primeros 60 días y una cantidad máxima para el tercer mes (Peng, 2011, p. 913; Lee et al, 2016, p. 275). 
hombres, ${ }^{31}$ las acciones afirmativas para mejorar el empleo y la instalación de infraestructura de cuidado infantil en el lugar de trabajo; teniendo en cuenta la situación actual, legislar sobre la reducción de las horas de trabajo para el cuidado infantil, el aumento del pago de beneficios durante la licencia de maternidad - que los países avanzados ni siquiera pagan- y la licencia de paternidad, definitivamente limitará la gestión de recursos humanos de una empresa y traerá más déficit al presupuesto del seguro de desempleo.

Funcionarios de la KEF añaden que hay otro programa público con una asignación familiar mensual de aproximadamente 100 dólares al mes para cada menor a seis años (KEF, comunicación personal, 9 de julio de 2019).

Poco después, en 2014, frente al "plan de apoyo para la administración del ciclo de vida de las trabajadoras", la KeF (2014a) insistió al reconocer "la necesidad de mejorar la participación femenina en la fuerza laboral y prevenir la discontinuidad de su carrera":

La licencia por cuidado infantil y la ampliación de la reducción de la jornada laboral para el cuidado infantil influyen enormemente en la gestión de recursos humanos de las empresas. Sin embargo, aumenta la preocupación de que las leyes gubernamentales de protección de la maternidad del Gobierno, incluido el plan de apoyo, podrían aumentar las cargas de las empresas relacionada con el empleo femenino $y$, por lo tanto, el plan reduciría el empleo femenino contra la intención original de las leyes.

Además, dado que las medidas señaladas se financiarían a través del seguro de desempleo, "podría generarse un gasto enorme al implementar dichas políticas contra la intención original del fondo [de desempleo] y dañarlo cuando ya está en déficit”.

31. Los padres, desde 2007 con la Ley de Asistencia para la Igualdad de Oportunidades en el Empleo y el Equilibrio entre el Trabajo y la Familia en 2007, pueden tomar ¡sólo cinco días de licencia por paternidad (tres días pagados, dos días no pagados)! Además, en 2008 se generó la posibilidad de que los empleados con hijos menores de tres años obtengan una reducción de su jornada de trabajo (15 a 30 horas por semana durante un año) (Lee, 2018, p. 51). Lee et al. (2016, pp. 275 y 277) señalan que esta posibilidad se amplió para padres con hijos menores de seis años, licencia parcialmente pagada con fondos del seguro del desempleo (40\% del salario mensual con límite en un millón de wones). Para Lee et al. (2016) estas posibilidades de licencia maternal y paternal son muy limitadas - podríamos llamar minimalistas - en comparación con países con instituciones de bienestar avanzadas como Suecia, además de que en Corea un porcentaje importante de madres y padres no utilizan estas licencias a cabalidad. 
Ante otras medidas relacionadas con la carrera laboral de las mujeres, diversas organizaciones también se pronunciaron para evitar cargas excesivas o para ampliar las posibilidades institucionales de cuidado. Desde 2013 se había generado una declaración conjunta de KEF, FKI, K-Biz, KCCI y KITA (KEF, 2013b) sobre diversos aspectos laborales y propuestas de políticas públicas, entre las que cuestionan que los "puntos extras para las madres [que desean regresar al trabajo después de un receso profesional debido al proceso de embarazo-parto-cuidado de niños] intensificarán la confusión en el mercado laboral ya que causa discriminación contra mujeres solteras y mujeres casadas sin hijos, y hombres".

Estas mismas organizaciones, más la Association of High Potencial Enterprises of Korea, se pronunciaron en favor de la dualización del sistema de cuidado infantil con atención de todo el día (12 horas) o de corta duración (seis horas), dado que la comunidad de negocios del cuidado infantil sólo prefiere estas últimas.

Las empresas crearemos un entorno que sea amigable para el parto y el cuidado infantil, incluido el avance de la cultura empresarial (como la mejora frente a la práctica de largas horas de trabajo y el trabajo habitual de horas extras), la expansión de las instalaciones de cuidado infantil en los lugares de trabajo y la expansión del sistema de horario flexible de trabajo (KEF, 2016b).

En esta línea de flexibilidad y apoyo al cuidado infantil, FKI dijo al Korea Herald (2015): "15 grupos empresariales de los 30 principales en términos de activos manifestaron que al menos una de sus filiales ha adoptado horarios de trabajo flexibles para sus empleados. Incluyen Samsung, SK, LG, Lotte y POSCO”. En algunos casos este cambio ha sido aplicado desigualmente sólo a los "trabajadores no productivos". "El sistema de trabajo flexible permite a los empleados elegir sus horas de trabajo diaria y semanalmente para que puedan manejar sus asuntos personales, como el cuidado de niños y las actividades de ocio".

Los empresarios estudiados reconocen "la gravedad de la baja tasa de natalidad sin precedentes como un desafío nacional", cuestionan la débil presencia del Estado en el cuidado infantil (sólo 7\% de guarderías públicas en el país en 2016, proporción muy baja en términos internacionales, comparada con el 85\% de Francia en 2013 y el 80\% de Suecia en 2011), señalan por otra parte que ya hay programas para fomentar el cuidado por parte de las madres y padres trabajadores, y proponen como solución una mayor flexibilidad 
laboral (KEF, comunicación personal, 9 de julio de 2019). En esta tensión entre reconocimiento y voz crítica, resalta que en parte los empresarios no solicitan simplemente menor presencia del Estado o privatizaciones: por un lado, pugnan por más guarderías públicas; por otro, sin embargo, cuestionan de nuevo las consideradas excesivas regulaciones públicas sobre el empleo. La prioridad, en estos asuntos del cuidado, sigue siendo priorizar el crecimiento con flexibilidad laboral.

\section{Conclusiones}

Lo que encontramos en el estudio de los anteriores posicionamientos en relación con los siete aspectos vinculados a las políticas de bienestar en Corea, es que en síntesis las organizaciones empresariales analizadas en el periodo 2009-2019 viven en una especie de contradicción permanente en relación con las instituciones de bienestar, que tienen institucionalmente una tendencia hacia la universalización (Fleckenstein \& Lee, 2017, p. 40; Valencia, 2019): por una parte, se manifiestan con "lealtad" a la Hirschman y participan en las instituciones con las contribuciones acordadas en los diversos seguros sociales (salud, pensiones, accidentes, cuidado de largo plazo), en las actividades de cuidado infantil (guarderías en las empresas); por otra, se manifiestan con tensiones dirigidas a la "salida" a la Hirschman, con amenazas de exportación o abandono de las inversiones ante la "excesiva" carga social de estos seguros, de deslocalización industrial e incluso de automatización (para evitar las presiones colectivas y de nuevas cargas sociales). La narrativa de exceso en los costos está continuamente presente, en el marco de un país con aún un reducido gasto social.

La presión hacia la "salida" se expresa en la resistencia de invertir, porque la inversión productiva implica a fin de cuentas asociarse a los acuerdos sociales empotrados en las instituciones sociales que crean sus propias inercias históricas (path dependence), generalmente con crecimiento de obligaciones y derechos. La tensión entre la "lealtad" y la "salida" se expresa también en una dominación de la "voz" a la Hirschman, voz crítica, con la que cuestionan los excesos de regulaciones, de costos sociales y protección laboral, las medidas que atentan contra la competitividad de las empresas, y proponen permanentemente la flexibilización de las relaciones laborales, la disminución de regulaciones y la disminución de costos laborales y sociales. Así, la "lealtad" por las instituciones de política social de Corea se ve tensionada por la bús- 
queda de reformas liberales con predominio de menor Estado ${ }^{32}$ más eficiente (menores cargas sociales), de la flexibilización de las relaciones laborales y de la meritocracia y responsabilidad individual (o sistema guiado por el desempeño de los trabajadores), aunque con menor presencia de dinámicas privatizadoras. ${ }^{33}$ La voz crítica sobre la política social de los empresarios organizados tiende a plegarse así a las históricas demandas o preferencias de la coalición pro-crecimiento o productivista coreana, y a relativizar las demandas redistributivas de la sociedad coreana.

De hecho, no encontramos ningún pronunciamiento explícito en favor de la redistribución, lo que por ausencia es ya un símbolo importante. No hay una voz crítica favorable a la universalización (con la excepción del seguro de salud) o a la extensión de derechos (caminos de redistribución), sino más bien dirigida hacia el freno o reducción de las "cargas" sociales. Entre la flexibilización liberal y la flexiseguridad, ${ }^{34}$ las organizaciones empresariales presionan hacia la primera, al menos en la narrativa, y muestran una inclinación hacia la coalición histórica pro-crecimiento en el régimen de bienestar coreano.

Si las élites socioeconómicas mostraban la tensión entre la visión distributiva y la meritocracia, con dominio probablemente de ésta, las organizaciones empresariales estudiadas manifestaban la tensión entre la lealtad a las instituciones de bienestar construidas durante el proceso reciente de desarrollo en Corea y una constante voz crítica desde el libre mercado, la flexibilidad laboral, la meritocracia, la competitividad y el crecimiento económico; voz crítica que conlleva la amenaza a la salida del juego económico de la propia Corea para deslocalizar inversiones en un espacio de menores costos sociales o simplemente para dejar de invertir en Corea. Sí hay propuestas explícitas de meritocracia (salarios ajustados por desempeño) y no las hay respecto de la redistribución. Así, encontramos una coherencia entre las preferencias de las élites socioeconómicas de la primera parte de este texto y las prioridades empresariales en las complejas tensiones entre lealtad, voz (crítica) y salida.

32. Con excepción de las guarderías, en relación con las que los empresarios entrevistados cuestionan la débil participación pública.

33. Presencia más abierta en la exigencia de mayores inversiones en hospitales privados.

34. Para Boyer (2006, p. 19), la flexiseguridad (se refiere al caso danés) incluye por un lado flexibilidad de las reglas de contratación y de despido, y por otro una cobertura universal con indemnizaciones de desempleo generosas y con una política activa de empleo centrada en la formación de los trabajadores. 
Esta complejidad en tensión permite verificar lo señalado en Valencia (2019, p. 193): "la novedad es que el piso de la discusión sobre bienestar ya no es el residualismo de acuerdo con los debates del inicio de la segunda década del siglo Xxi. Simbólicamente, el concepto bienestar ya no es sólo legítimo para la coalición redistributiva".

Estas tensiones se manifestaron en la entrevista en la sede de la KEF ante dos preguntas: primera, entre flexibilidad laboral y cobertura universal de las instituciones sociales, ¿qué prefieren las empresas? La respuesta expresó claramente la tensión y la preferencia entre valores: valoramos la cobertura universal, pero "realistamente lo más importante es la flexibilidad laboral". Segunda, en caso de tener que elegir entre "derechos sociales" y "sistema basado en el desempeño", ¿qué considerarían más importante? (KEF, comunicación personal, 9 de julio de 2019). De nuevo quedó establecida la tensión: los derechos sociales - que no fueron respetados durante el autoritarismo- son importantes pero la productividad y la eficiencia lo son más. De esta manera, si bien las organizaciones empresariales estudiadas no manifiestan una visión minimalista de las políticas sociales, no parecen situarse en la lógica de la coalición redistributiva y continúan en las preferencias centrales de la coalición pro-crecimiento.

¿Por qué esta insistencia empresarial en sostener la coalición pro-crecimiento y en resistir ante los procesos de redistribución? Responder a esta pregunta implica continuar profundizando la investigación, con la inclusión de otros actores y el análisis de las prácticas empresariales en terrenos de la creación de las políticas sociales. Al menos podemos aventurar algunas hipótesis: las organizaciones analizadas defienden con vehemencia los intereses de las empresas y consideran que éstas pueden ser más competitivas (y generadoras de utilidades) en contextos de flexibilidad laboral; ésta puede ser la primera hipótesis, de carácter utilitario; sin embargo, podría ser insuficiente para capturar la complejidad de los procesos institucionales e históricos en juego. Así, pueden plantearse otras dos explicaciones complementarias: por una parte, la fuerza de la inercia histórica (path dependence) que lleva a las organizaciones empresariales de los grandes conglomerados a defender las preferencias centrales de la coalición pro-crecimiento en contextos de conflictos sociales (laborales) y políticos; por otra, la ausencia de una dinámica hacia un gran acuerdo nacional pro-redistribución en la línea de algunos países socialdemócratas, a pesar de los avances innegables de las políticas sociales coreanas tendencialmente universalistas. La trayectoria próxima del campo 
conflictivo de las políticas sociales mostrará el resultado de las confrontaciones distributivas y la ubicación de las élites socioeconómicas.

\section{Referencias}

Boyer, R. (2006). La flexicurité danoise: quels enseignements pour la France? [Flexiguridad danesa ¿qué lecciones para Francia?]. Éditions Rue d'Ulm; Presses de l'École normale supérieure.

Boyer, R. (2014). Los mundos de la desigualdad. Un análisis a partir de la teoría de la regulación y una respuesta a Thomas Piketty. Editorial Octubre.

Choi, K. (2014, marzo 4). More firms mull retirement extension plans. The Korea Times.http://www.koreatimes.co.kr/www/tech/2018/12/693_152734.html

Choi, S. (2016, diciembre 8). Disband chaebol lobby. The Korea Times. https:// www.koreatimes.co.kr/www/opinion/2019/02/673_215861.html

Chung, H. (2012, junio 17). Corporations hoarding cash? The Korea Times. http://www.koreatimes.co.kr/www/biz/2019/06/488_113183.html

Cornia, G. A. (2016). The decline and recent uptick of income inequality in Latin America, 2002-13. En UNESCO e International Social Science Council, World Social Science Report, 2016: Challenging inequalities, pathways to a just world (pp. 201-205). UNESCO.

Esping-Andersen, G. (1990). The Three Worlds of Welfare Capitalism. Polity Press.

Esping-Andersen, G. (1999). Social Foundations of Postindustrial Economies. Oxford University Press.

Fiori, A., \& Kim, S. (2011). The Dynamics of Welfare Policy-Making in South Korea: Social Movements as Policy Entrepreneurs. Asian Social Work and Policy Review, 5(2), 61-78. https://doi.org/10.1111/j.17531411.2011.00053.x

FKI. (1999, febrero 11). The Charter of Business Ethics. FKI. http://www.fki. or.kr/en/About/Charter1.aspx

FKI. (2003 abril 21). FKI's Vision \& Mission. FKI. http://www.fki.or.kr/en/ About/Charter3.aspx

FKI. (2020). Who are the members of FKI? FKI. http://www.fki.or.kr/en/About/ fkiInfo.html\#3

Fleckenstein, T., \& Lee, S. Ch. (2017). Democratization, post-industrialization, and East Asian welfare capitalism: the politics of welfare state reform in 
Japan, South Korea, and Taiwan. Journal of International and Comparative Social Policy, 33(1), 36-54. https://doi.org/10.1080/21699763.2017.1288158 Hirschman, A. O. (1977). Salida, Voz y Lealtad. FCE.

Holliday, I. (2005). East Asian Social Policy in the Wake of the Financial Crisis: Farewall to Productivism. Policy and Politics, 33(1), 145-162. https://doi. org/10.1332/0305573052708465

Inglehart, R., Haerpfer, C., Moreno, A., Welzel, C., Kizilova, K., Diez-Medrano, J., Lagos, M., Norris, P., Ponarin, E., \& Puranen, B. et al. (Eds.). (2014). World Values Survey: Round Six - Country-Pooled Datafile Version. JD Systems Institute. http://www.worldvaluessurvey.org/WVSDocumentationWV6.jsp

Jun, I., \& Sheldon, P. (2006), Looking beyond the West? The Korea Employers' Federation and the Challenges of Membership Adhesion and Cohesion. The Economic and Labour Relations Review, 17(1), 203-225. https://doi. org $/ 10.1177 / 103530460601700108$

Jun, I., Sheldon, P., \& Lee, K. (2018). The Korea Employers' Federation and the development on Korean industrial relations. En Y. Lee \& B. E. Kaufman (Eds.), The Evolution of Korean Industrial and Employment Relations (pp. 63-82). Edward Elgar Publishing.

Kang, W. (2015). Inequality, the welfare system and satisfaction with democracy in South Korea. International Political Science Review, 36(5), 493-509. https://doi.org/10.1177\%2F0192512114521053

Kim, N. N. (2018). Wealth Inequality in Korea, 2000-2013. Evidence from Inheritance Tax Statistics. Journal of the Korean Welfare State and Social Policy, 2(1), 26-57.

Kim, R. (2013, agosto 25). Firms moving factories abroad. The Korea Times. http://www.koreatimes.co.kr/www/tech/2018/11/693_141667.html

Kim, T. (2009, julio 1). Employers Divided on Non-Regular Workers Law. The Korea Times. http://www.koreatimes.co.kr/www/news/nation/2009/07/117_47796.html

Kim T. (2011, mayo 16). Militant trade unions boost national competitiveness. The Korea Times. http://www.koreatimes.co.kr/www/ tech/2018/10/693_87078.html

Kim, Y. (2013, diciembre 22). Firms may boost factory relocations. The Korea Times. http://www.koreatimes.co.kr/www/tech/2019/04/133_148364.html

Kim, Y. (2015, marzo 22). FKI calls for regulatory easing. The Korea Times. http://www2.koreatimes.co.kr/www/tech/2019/05/133_175677.html 
Klasen, S., Scholl, N. Lahoti, R. Ochmann, S., \& Vollmer, S. (2016). InequalityWorldwide Trends and Current Debates (Discussion Papers 206). Courant Research Centre PEG.

Korea Enterprises Federation (KEF). (2009, octubre 13). Status of increasing social security cost for enterprise and policy directions. KEF. http://www. kefplaza.com/kef/kef_eng_intro_6_1.jsp?num=859\&pageNum=30

Korea Enterprises Federation (KEF). (2010, octubre 1). Business standpoint regarding the government's plan to deal with the low birthrate and aging society. KEF. http://www.kefplaza.com/kef/kef_eng_intro_6_1. jsp?num=1029\&pageNum=33

Korea Enterprises Federation (KEF). (2013a, julio 12). 2013 Research on Extension of the Retirement Age. KEF. http://www.kefplaza.com/kef/ kef_eng_intro_6_1.jsp?num=4890\&pageNum=20

Korea Enterprises Federation (KEF). (2013b, abril 30). Business standpoint on recent legislation about economic and labor issues. KEF. http://www.kefplaza. com/kef/kef_eng_intro_6_1.jsp?num=4750\&pageNum=21

Korea Enterprises Federation (KEF). (2013c, octubre 31). Experience in advanced countries to achieve $70 \%$ employment rate and its implications. KEF. http://www.kefplaza.com/kef/kef_eng_intro_6_1. jsp?num $=5269$ \&pageNum $=23$

Korea Enterprises Federation (KEF). (2014a, febrero 11). Business comment on the government's "lifecycle career management support plan for female workers". KEF. http://www.kefplaza.com/kef/kef_eng_intro_6_1. jsp?num=5589\&pageNum $=15$

Korea Enterprises Federation (KEF). (2014b, agosto 29). Current Status of Social Insurance Burden and Suggestions for Improvement. KEF. http://www. kefplaza.com/kef/kef_eng_intro_6_1.jsp?num=6069\&pageNum=17

Korea Enterprises Federation (KEF). (2014c, octubre 24). Business Standpoint on the measure to "Enhancing coverage of social insurance for parttime jobs". KEF. http://www.kefplaza.com/kef/kef_eng_intro_6_1. jsp?num=6289\&pageNum $=14$

Korea Enterprises Federation (KEF). (2015a, enero 1). 2015 New Year's Message from KEF. KEF. http://www.kefplaza.com/kef/kef_eng_intro_6_1. jsp?num $=6549$ \&pageNum $=16$

Korea Enterprises Federation (KEF). (2015b, febrero 2). Business Stance On The Government's Policy On Occupational Safety And Health In- 
novation. KEF. http://www.kefplaza.com/kef/kef_eng_intro_6_1. jsp?num $=6609$ \&pageNum $=10$

Korea Enterprises Federation (KEF). (2015c, junio 29). Business Stance On The Determination of Health Insurance Rate for 2016. KEF. http://www.kefplaza. com/kef/kef_eng_intro_6_1.jsp?num=6929\&pageNum=10

Korea Enterprises Federation (KEF). (2015d, septiembre 3). Business Stance On The Labor Reform. KEF. http://www.kefplaza.com/kef/kef_eng_intro_6_1. jsp?num=7209\&pageNum $=13$

Korea Enterprises Federation (KEF). (2015e, septiembre 21). Current Status of Social Insurance Cost and Suggestions for Improvement. KEF. http://www. kefplaza.com/kef/kef_eng_intro_6_1.jsp?num=7369\&pageNum=15

Korea Enterprises Federation KEF. (2015f, diciembre 8). Research on Features and Current Situation of Wage Peak Systems. KEF. http://www.kefplaza. com/kef/kef_eng_intro_6_1.jsp?num=7649\&pageNum=12

Korea Enterprises Federation (KEF). (2015g, diciembre 16). Declaration of Business Actions to Overcome Low Birth Rate. KEF. http://www.kefplaza. com/kef/kef_eng_intro_6_1.jsp?num=7650\&pageNum $=10$

Korea Enterprises Federation (KEF). (2016a, enero 1). 2016 New Year's Message from KEF. KEF. http://www.kefplaza.com/kef/kef_eng_intro_6_1. jsp?num $=7729$ \&pageNum $=5$

Korea Enterprises Federation (KEF). (2016b, junio 24). Business Stance on the Implementation of Customized Childcare to Overcome an Era of Low Growth. KEF. http://www.kefplaza.com/kef/kef_eng_intro_6_1. jsp?num $=8250$ \&pageNum $=4$

Korea Enterprises Federation (KEF). (2016c, octubre 17). Current Status of Social Insurance Cost and Suggestions for Improvement. KEF. http://www. kefplaza.com/kef/kef_eng_intro_6_1.jsp?num=8669\&pageNum=0

Korea Enterprises Federation (KEF). (2017a, marzo 27). KEF Survey on the Changes in Personnel Management and Wage Systems since the Legislation of Retirement Age to 60. KEF. http://www.kefplaza.com/kef/kef_eng_intro_6_1.jsp?num=93898pageNum=6

Korea Enterprises Federation (KEF). (2017b, junio 23). Business Stance on the Introduction of Industrial Accident Insurance to Commuting Accidents. KEF. http://www.kefplaza.com/kef/kef_eng_intro_6_1. jsp?num $=9729 \&$ pageNum $=5$ 
Korea Enterprises Federation (KEF). (2017c, Julio 19). Business Stance on the Determination of the Minimum Wage for 2018. KEF. http://www.kefplaza. com/kef/kef_eng_intro_6_1.jsp?num=9689\&pageNum=0

Korea Enterprises Federation (KEF). (2017d, agosto 21). Business Stance on the New Administration's Preventive Measures on Serious Industrial Accidents. KEF. http://www.kefplaza.com/kef/kef_eng_intro_6_1. jsp?num $=9849 \&$ \&ageNum $=5$

Korea Enterprises Federation (KEF). (2018a, enero 1). 2018 New Year's Message from KEF. KEF. http://www.kefplaza.com/kef/kef_eng_intro_6_1. jsp?num $=10332 \&$ pageNum $=4$

Korea Enterprises Federation (KEF). (2018b, marzo 12). Inauguration speech by the 7th KEF chairman. KEF. http://www.kefplaza.com/kef/kef_eng_intro_6_1.jsp?num=10610\&pageNum=4

Korea Enterprises Federation (KEF). (2018c, abril 13). "Opinion of management about the entire amendment bill of the Industrial Safety Health Act" submitted by the KEF. KEF. http://www.kefplaza.com/kef/kef_eng_intro_6_1. jsp?num $=10629$ \&pageNum $=3$

Korea Enterprises Federation (KEF). (2018d, abril 19). Business Stance on Disclosing Safety \& Health Data. KEF. http://www.kefplaza.com/kef/ kef_eng_intro_6_1.jsp?num=10649\&pageNum=3

Korea Enterprises Federation (KEF). (2018e, julio 4). KEF made a Historic Turning Point at its Extraordinary General Meeting. KEF. http://www.kefplaza. com/kef/kef_eng_intro_6_1.jsp?num=10929\&pageNum=3

Korea Enterprises Federation (KEF). (2018f, Julio 23). KEF objects the Reported Minimum Wage for 2019. KEF. http://www.kefplaza.com/kef/kef_eng_intro_6_1.jsp?num=11069\&pageNum=2

Korea Enterprises Federation (KEF). (2018g, noviembre 6). Business Stance on the raising the Long-term Care Insurance for 2019. KEF. http://www. kefplaza.com/kef/kef_eng_intro_6_1.jsp?num=11889\&pageNum=1

Korea Enterprises Federation (KEF). (2018h, diciembre 10). KEF Submitted Employers' Statement on Eight Key Bills to the National Assembly. KEF. http://www.kefplaza.com/kef/kef_eng_intro_6_1. jsp?num=12109\&pageNum $=1$

Korea Enterprises Federation (KEF). (2018i, diciembre 17). Employers Strongly Oppose the Government's Amendment of the Minimum Wage Enforcement Decree. KEF. http://www.kefplaza.com/kef/kef_eng_intro_6_1. jsp?num=12209\&pageNum $=1$ 
Korea Enterprises Federation (KEF). (2018j, diciembre 24). KEF announced "2017 Social Insurance Contribution Borne by the Nation". KEF. http://www. kefplaza.com/kef/kef_eng_intro_6_1.jsp?num=12169\&pageNum=1

Korea Enterprises Federation (KEF). (2019, abril 10). Business comments on Ministry of Health and Welfare's proposal on the 1st Comprehensive National Health Insurance Plan. KEF. http://www.kefplaza.com/kef/kef_eng_intro_6_1.jsp?num $=12629 \&$ pageNum $=0$

Korea Enterprises Federation (KEF). (2020a). Chairman's Remarks. KEF. http://www.kefplaza.com/kef/kef_eng_intro_1.jsp

Korea Enterprises Federation (KEF). (2020b). KEF Wage Peak Model. KEF. KEF. http://www.kefplaza.com/kef/labor_infocus_view.jsp?num=964

Korea Enterprises Federation (KEF). (2020c). Services and Offers. KEF. http:// www.kefplaza.com/kef/kef_eng_intro_9.jsp

Korea Herald. (2015, julio 13). Biz groups urged to adopt flexible working system. Korea Herald. http://heraldk.com/en/2015/07/13/biz-groupsurged-to-adopt-flexible-working-system/

Korea Labor Institute (KLI). (2017). 2017 IV Working Life. KLI Labor Statistics Archive. https://www.kli.re.kr/kli_eng/selectBbsNttView.do?key=381\& bbsNo=35\&nttNo=131409\&searchY=\&search Ctgry=\&searchDplcCtgry $=\&$ searchCnd=all\&searchKrwd=\&pageIndex=1\&integrDeptCode $=$

Korea Times. (2016a, mayo 10). FKI presses for seven regulatory reforms to create jobs. The Korea Times. https://www.koreatimes.co.kr/www/ tech/2020/07/129_204369.html

Korea Times. (2016b, junio 26). S. Koreans workers' productivity drops in last 5 years: FKI survey. The Korea Times. https://www.koreatimes.co.kr/ www/nation/2018/12/113_207879.html

Lee, D. (2018). The evolution of family policy in South Korea: From Confucian familism to Neo-familism. Asian Social Work and Policy Review, 12(1), 46-53. https://doi.org/10.1111/aswp.12137

Lee, H. (2015a, junio 29). Baby boomers urged to share fruits with young. The Korea Times. http://www.koreatimes.co.kr/www/ tech/2019/03/693_181821.html

Lee, H. (2015b, agosto 31). Businesses demand easier dismissals. The Korea Times. http://www.koreatimes.co.kr/www/tech/2019/06/693_185898.html Lee, H. (2017, abril 30). Firms feared to leave Korea due to high taxes. The Korea Times.https://www.koreatimes.co.kr/www/biz/2020/02/602_228524.html 
Lee, K. (2020, junio 29). Minimum wage discussion to strain businesses. The Korea Times. http://www.koreatimes.co.kr/www/nation/2020/07/367_291998.html

Lee, S., Duvander, A. Z., \& Zarit, S.H. (2016). How can family policies reconcile fertility and women's employment? Comparisons between South Korea and Sweden. Asian Journal of Women's Studies, 22(3), 269-288. https:// doi.org/10.1080/12259276.2016.1202027

Peng, I. (2011). The Good, the Bad and the Confusing: The Political Economy of Social Care Expansion in South Korea. Development and Change, 42(4), 905-923. https://doi.org/10.1111/j.1467-7660.2011.01724.x

Piketty, T. (2013). Le capital au XXIe siècle [El Capital en el Siglo XXI]. Éditions du Seuil.

Sabatier, P. A., \& y Weible. Ch. M. (2007). The Advocacy Coalition Framework. Innovations and Clarifications. En P. A. Sabatier (Ed.), Theories of the Policy Process (pp. 189-220). Westview Press.

Shin, D. (2003). Social and Economic Policies in Korea. Ideas, Networks and Linkages. Routledge. https://doi.org/10.4324/9780203449967

Sun, H. (2002). The political economy of Democratic Consolidation. Dynamic Labour Policy in South Korea. Chonnam National University Press.

The Social Science Research Institute. (2019). ISSP-International Social Survey Programme 2009. https://fel.hi.is/issp_international_social_survey_programme_0

Valencia, E. (1996). Deux ajustements, deux trajectoires: les politiques d'ajustement de la Corée du Sud et du Mexique (1979-1992) [Tesis de Doctorado, Universidad de París VII].

Valencia, E. (2009). El debate sobre los regímenes de bienestar en el este de Asia. El caso de Corea del Sur. En C. Barba, G. Ordóñez-Barba \& E. Valencia Lomelí (Coords.), Más allá de la pobreza. Regímenes de bienestar en Europa, Asia y América (pp. 291-324). El Colegio de la Frontera Norte; Universidad de Guadalajara.

Valencia, E. (2019). Coaliciones y transformación del régimen de bienestar en Corea del Sur (1987-2011). En J. L. Calva (Coord.). Trampas de desigualdad y cambios de régimen (pp. 167-197). Juan Pablos Editor; Consejo Nacional de Universitarios.

Yoon, J. (2016, mayo 4). Business lobby pushes for shorter work hours. The Korea Times.http://www.koreatimes.co.kr/www/tech/2018/11/693_204084.html 